\title{
Engineered type 1 regulatory T cells designed for clinical use kill primary pediatric acute myeloid leukemia cells
}

Haematologica 2021

Volume 106(10):2588-2597

\section{Correspondence:}

MARIA GRAZIA RONCAROLO mg1@stanford.edu

Received: June 15, 2020.

Accepted: September 17, 2020.

Pre-published: September 28, 2020.

https://doi.org/10.3324/haematol.2020.263129

(C)2021 Ferrata Storti Foundation

Material published in Haematologica is covered by copyright. All rights are reserved to the Ferrata Storti Foundation. Use of published material is allowed under the following terms and conditions:

https://creativecommons.org/licenses/by-nc/4.0/legalcode. Copies of published material are allowed for personal or internal use. Sharing published material for non-commercial purposes is subject to the following conditions:

https://creativecommons.org/licenses/by-nc/4.0/legalcode, sect. 3. Reproducing and sharing published material for commercial purposes is not allowed without permission in writing from the publisher.

\author{
Brandon Cieniewicz, ${ }^{1 *}$ Molly Javier Uyeda, ${ }^{1,2^{*}}$ Ping (Pauline) Chen, ${ }^{1}$ \\ Ece Canan Sayitoglu, ${ }^{1}$ Jeffrey Mao-Hwa Liu, ${ }^{1}$ Grazia Andolfi, ${ }^{3}$ \\ Katharine Greenthal, ${ }^{1}$ Alice Bertaina, ${ }^{1,4}$ Silvia Gregori, ${ }^{3}$ Rosa Bacchetta, ${ }^{1,4}$ \\ Norman James Lacayo, ${ }^{1}$ Alma-Martina Cepika ${ }^{1,4 *}$ \\ and Maria Grazia Roncarolo ${ }^{1,2,4,}$
}

${ }^{1}$ Department of Pediatrics, Division of Stem Cell Transplantation and Regenerative Medicine, Stanford School of Medicine, Stanford, CA, USA; ${ }^{2}$ Stanford Institute for Stem Cell Biology and Regenerative Medicine, Stanford School of Medicine, Stanford, CA, USA; ${ }^{3}$ San Raffaele Telethon Institute for Gene Therapy, Milan, Italy and ${ }^{4}$ Center for Definitive and Curative Medicine, Stanford School of Medicine, Stanford, CA, USA

${ }^{\star} B C$ and $M J U$ contributed equally as co-first authors

${ }^{\#} A M C$ and MGR contributed equally as co-senior authors

\section{ABSTRACT}

- Wpe 1 regulatory (Tr1) T cells induced by enforced expression of interleukin-10 (LV-10) are being developed as a novel treatment for chemotherapy-resistant myeloid leukemias. In vivo, LV-10 cells do not cause graft-versus-host disease while mediating graft-versus-leukemia effect against adult acute myeloid leukemia (AML). Since pediatric AML (pAML) and adult AML are different on a genetic and epigenetic level, we investigate herein whether LV-10 cells also efficiently kill pAML cells. We show that the majority of primary pAML are killed by LV-10 cells, with different levels of sensitivity to killing. Transcriptionally, pAML sensitive to LV-10 killing expressed a myeloid maturation signature. Overlaying the signatures of sensitive and resistant pAML onto the public NCI TARGET pAML dataset revealed that sensitive pAML clustered with M5 monocytic pAML and pAML with MLL rearrangement. Resistant pAML clustered with myelomonocytic leukemias and those bearing the core binding factor translocations inv(16) or $t(8 ; 21)$ (RUNX1RUNX1T1). Furthermore, resistant pAML upregulated the membrane glycoprotein CD200, which binds to the inhibitory receptor CD200R1 on LV-10 cells. In order to examine if CD200 expression on target cells can impair LV-10 cell function, we overexpressed CD200 in myeloid leukemia cell lines ordinarily sensitive to LV-10 killing. Indeed, LV-10 cells degranulated less and killed fewer CD200-overexpressing cells compared to controls, indicating that pAML can utilize CD200 expression for immune evasion. Altogether, the majority of pAML are killed by LV-10 cells in vitro, supporting further LV-10 cell development as an innovative cell therapy for pAML.

\section{Introduction}

Pediatric acute myeloid leukemia (pAML) affects over 700 children in the US every year. While pAML comprises only $25 \%$ of all pediatric acute leukemias, it accounts for almost half of pediatric leukemia-related deaths. Five-year survival rates for pAML have risen to over $60 \%$, in part due to improved risk-stratification, supportive care, and post-relapse treatment. ${ }^{1}$ However, between $30-55 \%$ of patients eventually relapse, ${ }^{2,3}$ and relapse remains the most frequent cause of death. Current treatment for relapsed or treatment-refractory pAML is allogeneic hematopoietic stem cell transplantation (allo-HSCT). ${ }^{4}$ Unfortunately, allo-HSCT carries the significant risk of inducing life-threatening graft-versus-host disease (GvHD) mediated by donorderived T cells. GvHD is the major cause of transplant-related morbidity and mortality, and the second leading cause of death in AML patients. ${ }^{5,6} \mathrm{GvHD}$ can be treated 
with immunosuppressive drugs, but these treatments also impair donor-derived cells from clearing residual leukemia cells (graft-versus-leukemia effect [GvL]), ${ }^{9}$ thereby increasing the risk of relapse. Thus, new treatments that preserve GvL while preventing GvHD are urgently needed.

In order to address this need, we devised a novel cell therapy with engineered type 1 regulatory $\mathrm{T}$ ( $\operatorname{Tr} 1)$ cells, called LV-10, made by lentiviral transduction of interleukin-10 (IL10) into peripheral CD4 ${ }^{+} \mathrm{T}$ cells. Tr1 cells are a FOXP3subset of peripherally inducible regulatory $\mathrm{T}$ cells ${ }^{8}$ that correlate with induction of peripheral tolerance in transplanted patients $^{9-12}$ and prevent xeno-GvHD in mice. ${ }^{13-16}$ In addition, Tr1 cells directly lyse and kill malignant myeloid cells via perforin and granzyme B. ${ }^{13,14,17}$ This killing is not dependent on T-cell receptor (TCR) engagement ${ }^{13,18}$ and human leukocyte antigen (HLA) class II antigen presentation, but rather on the target cell expression of HLA class I and several other molecules that facilitate target cell and $\mathrm{T}$ cell interaction. ${ }^{13,14}$ Importantly, LV-10 Tr1 cells were shown to kill primary adult AML blasts and to impair leukemia progression in humanized mouse models of AML. ${ }^{13}$

The sensitivity of pAML to Tr1-mediated killing has not been tested. pAML have significant genetic, epigenetic, and molecular differences in comparison to adult AML. ${ }^{19-23}$ Understanding if pAML are also sensitive to Tr1-mediated killing is thus a critical step in LV-10 cell therapy development. Herein, we used LV-10 Tr1 cells to test 23 primary pAML blasts for their sensitivity to killing. ${ }^{13,14}$ We found that over $80 \%$ of pAML could be killed by LV-10 cells, with three levels of sensitivity to killing ranging from sensitive, intermediate resistant, and resistant. Sensitive pAML were enriched for gene signatures of leukocyte chemotaxis and expressed mature myeloid markers including CD64 and CD11c, suggesting a more mature phenotype. When analyzed together with the large National Cancer Institute (NCI) Therapeutically Applicable Research to Generate Effective Treatments (TARGET) sensitive pAML dataset, pAML formed three clusters with TARGET samples, including one enriched for sensitive pAML samples with French-American-British (FAB) M5 acute monocytic leukemia and pAML with mixed lineage leukemia (MLL) rearrangement, while resistant and intermediate resistant pAML clustered with pAML bearing core binding factor translocations inv(16) or t(8;21)(RUNX1-RUNX1T1) cytogenetic abnormalities. In addition, we identified that resistant pAML may evade LV-10 killing by upregulating CD200, which has been associated with poor prognosis of adult AML. ${ }^{24,25}$ Overall, we determine that a majority of pAML are sensitive to killing by LV-10 cells, and that resistance to killing is associated with loss of a mature myeloid signature and upregulation of CD200.

\section{Methods}

\section{Subjects}

De-identified pAML bone marrow aspirates were collected under written informed consent as part of a study approved by the Stanford University Institutional Review Board (IRB, \#11062 and \#11977) and obtained from the Stanford School of Medicine's Bass Childhood Cancer Center (CA, USA) tissue bank. Patient demographics are listed in Table 1. Human peripheral blood mononuclear cells (PBMC) were obtained from de-identified healthy donors (Stanford Blood Center, CA, USA) in accordance with IRB guidelines.
Table 1. Patient characteristics.

\begin{tabular}{lc} 
Age (months) (at sample acquisition) & 137 (5-267) \\
\hline \% Female & $43.50 \%$ \\
Average white blood cell count & $83.7 \mathrm{~K} / \mu \mathrm{L}(0.4-347.6)$ \\
\hline Risk stratification & \\
High risk & $12 / 23$ \\
Standard risk & $5 / 23$ \\
Low risk & $6 / 23$ \\
Average time of follow-up (months) & 33.5 \\
\hline Progression to HSCT (\%) & 43.50 \\
Overall survival (\%) & 73.90 \\
\hline
\end{tabular}

HSCT: hematopoietic stem cell transplantation.

\section{Cytotoxicity assays}

Killing assay was performed as previously described. ${ }^{13,14}$ Briefly, target cells were co-cultured at a 1:1 effector to target (E:T) ratio for 3 days. For primary pAML, blasts were thawed and incubated for 2 hours (h) in complete X-VIVO15 supplemented with IL-3 (20 $\mathrm{ng} / \mathrm{mL}$, Peprotech, NJ, USA) and G-CSF (20 ng/mL, Peprotech). After incubation, blasts were co-cultured for 4 days. Surviving cells were enumerated by fluorescence-activated cell sorting (FACS) using CountBright beads (Thermo Fisher, MA, USA). \%

Elimination efficiency equals 1 minus (number of targets remaining in LV-10 co-culture/number of targets remaining alone) *100 with two to four LV cell lines per pAML.

Degranulation was measured as previously described. ${ }^{13,14}$ Briefly, T cells were co-cultured with target cells at a 10:1 E:T ratio with anti-CD107a antibody. After $1 \mathrm{~h}$, brefeldin $\mathrm{A}(3 \mu \mathrm{g} / \mathrm{mL})$ and monensin $(2 \mu \mathrm{M})$ (eBioscience, CA, USA) were added and incubated for $5 \mathrm{~h}$. Cells were stained for surface markers, fixed, permeabilized (BD Fixation/Permeabilization kit, BD Biosciences), and stained for intracellular granzyme B as described in the Online Supplementary Table S1. Data was analyzed by flow cytometry. For CD200R1 blocking, $25 \mathrm{ug} / \mathrm{mL}$ of CD200R1 or isotype antibody was added to $\mathrm{T}$ cells for 30 minutes at $37^{\circ} \mathrm{C}$ prior to co-culturing with targets.

\section{RNA sequencing}

Complete computational methods for RNA sequencing (RNASeq) processing, analysis, and raw data are available at Gene Expression Omnibus (GEO) under accession number GSE140960. For differential gene expression, differential gene expression analysis (DESeq2) was used to normalize the counts and perform exploratory analysis (e.g., clustering, principal component analysis). ${ }^{26}$ Genes with low expression across all samples, sum (gene) $<10$ reads, were filtered out before performing differential gene expression. The design matrix was defined as design $=\sim$ condition, where the condition variable was composed of the following three levels: sensitive, intermediate resistant, and resistant. Transcripts were hierarchically clustered using Euclidean distance and complete linkage function. The heatmaps were created using ComplexHeatmap v2.0.0. ${ }^{27}$ Gene ontology (GO) terms were collapsed using EnrichmentMap v3.2.1 ${ }^{28}$ in Cytoscape v3.8.0. Correlation graph was plotted in $\mathrm{R}$ version 4.0.0. Enrichment analysis was performed using a binomial test for a one-tailed $P$-value, and Confidence Interval (CI) was calculated using Wilson/Brown test.

\section{Statistical analysis}

For the non-RNA-seq-derived data, analysis was performed using GraphPad Prism 7. As applicable, center bars and whiskers represent the mean with standard deviation, or median with range/interquartile range. The data was analyzed using non-para- 
Table 2. Pediatric acute myeloid leukemia patient clinical characteristics

\begin{tabular}{|c|c|c|c|c|c|c|c|c|c|c|}
\hline $\begin{array}{l}\text { AML } \\
\text { ID }\end{array}$ & $\begin{array}{l}\text { Response to } \\
\text { LV-10 }\end{array}$ & $\begin{array}{l}\text { Sample } \\
\text { Timepoint }\end{array}$ & $\begin{array}{c}\text { WHO } \\
\text { Classification }\end{array}$ & $\begin{array}{l}\text { FAB } \\
\text { Sulbtype }\end{array}$ & $\begin{array}{l}\text { Cytogenetics } \\
\text { Onset }\end{array}$ & $\begin{array}{c}\% \\
\text { Blast }\end{array}$ & $\begin{array}{c}\text { WBC } \\
\left(10^{3} / \mathrm{mL}\right)\end{array}$ & $\begin{array}{l}\text { Age } \\
\text { (M) }\end{array}$ & $\begin{array}{l}\text { Risk } \\
\text { group }\end{array}$ & MRD \\
\hline 3209 & Sensitive & Onset & AML - NOS & M5a & 46, XY, t(X;11)(5'MLL+) & 97 & 347.6 & 5 & $\mathrm{H}$ & - \\
\hline 186 & & Relapse & AML - NOS & M5a & 46, XY, MLL+ & 88 & 51.6 & 156 & $\mathrm{H}$ & + \\
\hline 646 & & Relapse & AML with MDS-related & M4Eo & 46, XY, del(7q)(q22) & 74 & 177 & 267 & $\mathrm{H}$ & + \\
\hline 3281 & & Onset & AML - NOS & M5b & $46, X X$ & 91 & 8.7 & 215 & $\mathrm{H}$ & - \\
\hline 3514 & & Onset & AML - NOS & M2 & $51, \mathrm{XY},+\mathrm{X},+9,+11,+14,+20$ & $n / a$ & 4 & 157 & S & + \\
\hline 335 & & Onset & AML - NOS & M5a & 46, XY, FLT3-ITD+ & 89 & 174 & 35 & $\mathrm{H}$ & - \\
\hline 263 & & Onset & AML with mutated RUNX1 & AML w/ MDS & $46, X Y$ & 61 & 9.8 & 141 & $\mathrm{H}$ & + \\
\hline 612 & & Onset & t(8;21); RUNX1-RUNX1T1 & M2 & $46, \mathrm{XY}, \mathrm{t}(8 ; 21)$ & 50 & 39.9 & 205 & L & - \\
\hline 3491.1 & $\begin{array}{l}\text { Intermediate } \\
\text { Resistant }\end{array}$ & Onset & APL with PML-RARA & M3 & $46, \mathrm{XX}, \mathrm{t}(15 ; 17)$ & 78 & 4.4 & 138 & S & - \\
\hline 3123 & & Relapse & MPAL & MPAL & 46, XX, Complex karyotype & 81 & 102 & 134 & $\mathrm{H}$ & + \\
\hline 1355 & & Onset & APL with PML-RARA & M3 & $46, \mathrm{XY}, \mathrm{t}(15 ; 17)$ & 75 & 2.1 & 119 & S & - \\
\hline 794 & & Onset & Inv(16); CBFB-MYH11 & M2 & $46, X X, \operatorname{Inv}(16)$ & 89 & 43 & 28 & $\mathrm{~L}$ & + \\
\hline 683 & & Onset & $\operatorname{Inv}(16) ;$ CBFB-MYH11 & M4Eo & 46, XY, Inv(16) & 89 & 56.1 & 190 & $\mathrm{~L}$ & - \\
\hline 882 & & Onset & MPAL & MPAL & 46, XY, t(7;14)(q21;q32) & 72 & 180.1 & 176 & $\mathrm{H}$ & + \\
\hline 351 & & Onset & $\begin{array}{l}\mathrm{t}(8 ; 21) ; \text { RUNX1-RUNX1T1, } \\
\text { trisomy } 21\end{array}$ & M2 & $47, \mathrm{XX}, \mathrm{t}(8 ; 21),+21$ & 75 & 0.4 & 194 & S & + \\
\hline 1244 & & Onset & $\begin{array}{c}\text { AML with mutated RUNX1, } \\
\text { trisomy } 21\end{array}$ & M7 & $48, X Y,+21,+Y$ & 50 & 37 & 14 & $\mathrm{H}$ & + \\
\hline 1563 & & Onset & t(8;21); RUNX1-RUNXIT1 & M2 & $46, \mathrm{XX}, \mathrm{t}(8 ; 21)$ & 61 & 51.3 & 178 & $\mathrm{~L}$ & + \\
\hline 3424 & & Relapse & t(9;11); MLLT3-KMT2A & M5a & $46, \mathrm{XX}, \mathrm{t}(9 ; 11)$ & $n / a$ & 2.8 & 162 & S & + \\
\hline 948 & & Onset & AML with mutated NPM1 & M2 & 46, XX, Complex karyotype & 82 & 153.7 & 51 & $\mathrm{H}$ & - \\
\hline 728 & Resistant & Onset & AML - NOS & M1 & $\begin{array}{c}\text { 46, XY, } \\
\mathrm{t}(1 ; 13)(\mathrm{p} 34 \sim 36 ; \mathrm{q} 13 \sim 14)[19]\end{array}$ & 77 & 3.9 & 207 & $\mathrm{H}$ & + \\
\hline 3082 & & Relapse & $\operatorname{Inv(16);~CBFB-MYH11~}$ & M4Eo & 46, XX, Inv(16) & 36 & 283 & 37 & $\mathrm{~L}$ & - \\
\hline 758 & & Onset & $\operatorname{Inv}(16) ;$ CBFB-MYH11 & M4E0 & $46, X X, \operatorname{Inv}(16)$ & 66 & 2.7 & 198 & $\mathrm{~L}$ & - \\
\hline 258 & & Onset & MPAL & MPAL & $46, X Y$ & 86 & 190 & 144 & $\mathrm{H}$ & - \\
\hline
\end{tabular}

AML: acute myeloid leukemia; MRD: minimal residual disease after first induction chemotherapy; WBC: white blood cell; H: high; S: standard; L: low risk group. Pediatric acute myeloid leukemia (pAML) samples were grouped based on their sensitivity to LV-10-mediated killing.Sample timepoint, World Health Organisation (WHO) classification, FrenchAmerican-English (FAB) classification, cytogenetics, blast percentage, white blood cell (WBC) count at diagnosis, age in months, risk group stratification, and minimal residual disease (MRD) status after first induction chemotherapy are displayed.

metric tests that do not assume equal variances between groups: Mann-Whitney or Wilcoxon test for groups of two (unpaired or paired samples, respectively), and Kruskal-Wallis or Friedman ANOVA with Dunn's post hoc test for $>2$ groups (independent or dependent samples, respectively). Multiple testing correction was applied. Linear regressions were plotted using linear regression analysis in GraphPad Prism.

\section{Results}

\section{Pediatric acute myeloid leukemia blasts have different levels of sensitivity to LV-10 killing}

In order to determine if pAML can be killed by LV-10 cells, we first generated LV-10 cells from healthy donorderived $\mathrm{CD} 4^{+} \mathrm{T}$ cells as described ${ }^{13,14}$ and verified their transduction efficiency, purity, cytokine profile, and killing capacity (Online Supplementary Figure S1). LV-10 cells had high transduction efficiency, high IL-10 and low IL-4, as well as high intracellular granzyme B expression at baseline (Online Supplementary Figure S1A to $E$ ) in comparison with effector T cell (Teff)-like control LV-GFP cells. LV-10 degranulation against target cells was also higher than LV-GFP cells, especially against HLA-class I positive myeloid tumor cell lines U937 and ALL-CM (Online Supplementary Figure S1F). LV-10 cells were able to potently eliminate U937 and ALL-CM cells, but not HLA-class I negative erythroleukemic K562 cell line (Online Supplementary Figure $S 1 G)$. Target cell elimination was also observed in control LV-GFP cells, which are not tolerogenic ${ }^{13}$ and thus are not further explored for clinical use.

Next, we tested if LV-10 cells could eliminate pAML. We obtained 23 pAML bone marrow aspirates, 18 at onset and five at relapse, of various World Health Organization $(\mathrm{WHO})^{29}$ and FAB diagnoses (Table 2). Killing-sensitive U937 and killing-resistant K562 cells were used as positive and negative controls, respectively. In the killing assay (see Materials and Methods), we observed three levels of pAML sensitivity to LV-10 killing: sensitive (S, >70\% median elimination efficiency [E.E.]), intermediate resistant (IR, 25-70\% median E.E.), and resistant ( $R,<25 \%$ median E.E.) (Figure $1 \mathrm{~A}$ and $\mathrm{B})$. Sensitivity or resistance was retested in nine pAML samples, and sensitivity levels were reproducible (not shown). Notably, all the pAML tested had high levels of HLA class I (not shown).

Because primary pAML typically expand poorly in vitro and can undergo spontaneous apoptosis, we examined if sensitivity correlated with pAML survival when cultured 


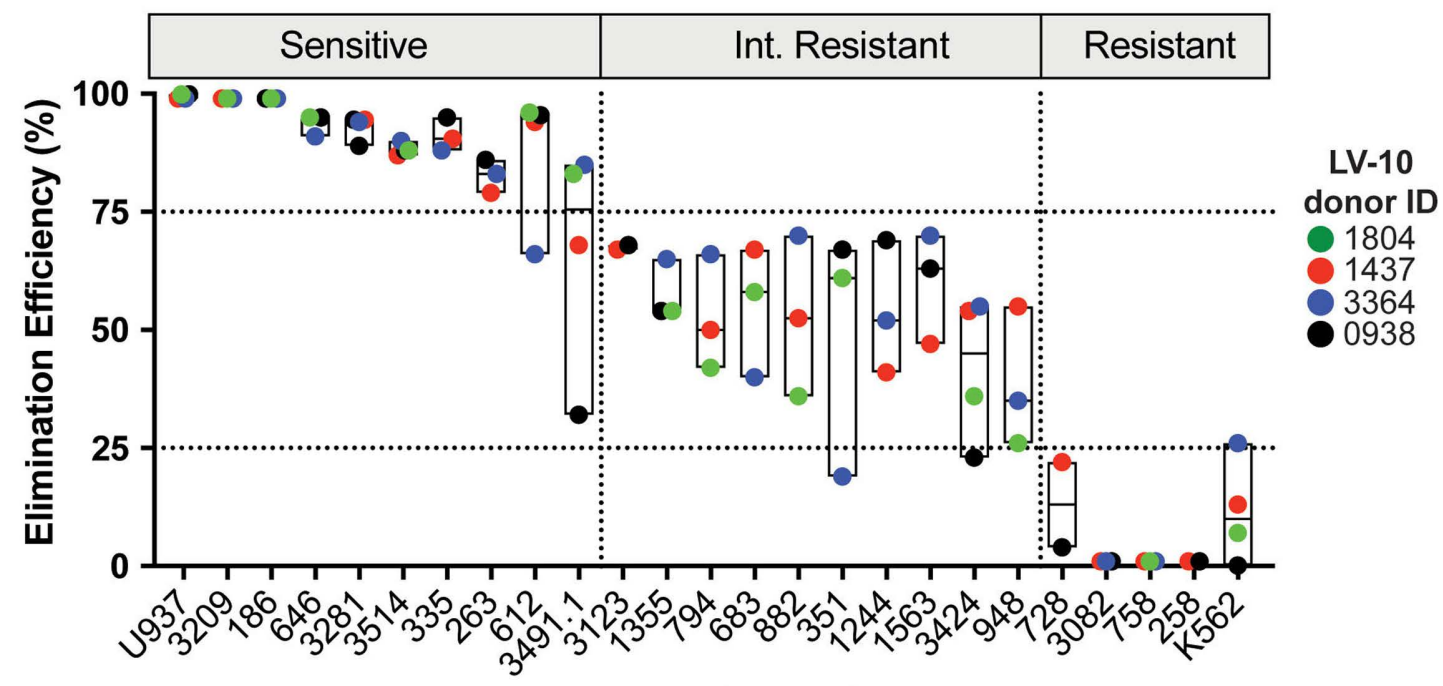

AML blast ID and control cell lines

\section{B Representative plots of remaining pAML after co-culture}
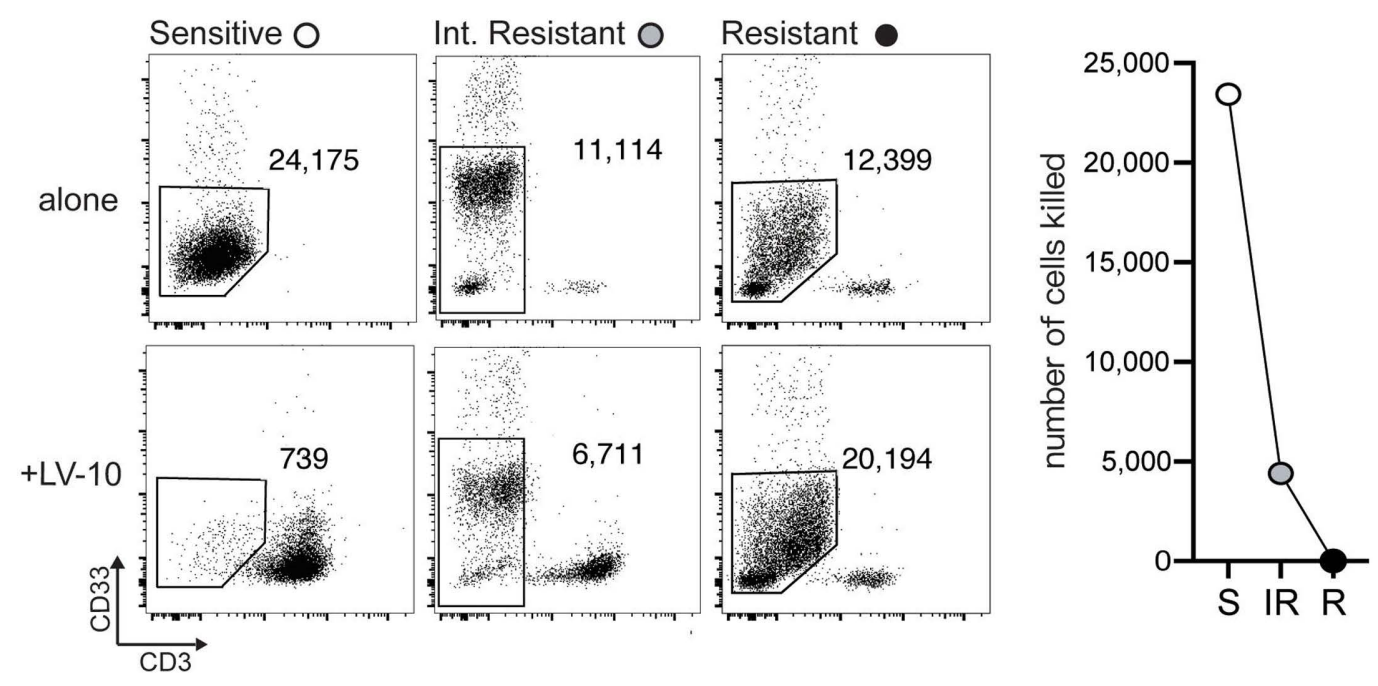

Figure 1. Pediatric acute myeloid leukemia have three levels of sensitivity to killing by LV-10 cells. (A) Primary pediatric acute myeloid leukemia (pAML) bone marrow aspirates were co-cultured at a 1:1 ratio with LV-10 cells. After 4 days, residual pAML (CD3-) were enumerated by flow cytometry (killing assay). Elimination efficiency (E.E.) was calculated for each LV-10 using the equation 1 - (AML remaining in LV-10 co-culture/AML remaining alone). U937 and K562 cells were included as positive and negative controls, respectively, for killing. The solid line indicates the median elimination efficiency, while the box boundaries indicate the range. Each dot represents the E.E. determined by co-culture with one LV-10 cell line, $n=2-4$. (B) Left panel contains representative plots of remaining pAML after the killing assay with or without LV-10; numbers on plots indicate the number of pAML cells normalized to the CountBright beads. Gating is set based on pAML cells cultured alone. Right panel graphs the representative examples to show the absolute differences between the number of pAML cells cultured with LV-10 and alone. S: sensitive; IR (or Int. Resistant): intermediate resistant; R: resistant.

without LV-10 cells. Survival of pAML cultured in medium alone did not correlate with their sensitivity to killing when cultured with LV-10 (Online Supplementary Figure S2A). pAML sensitivity to killing also did not correlate with blast percentage within the bone marrow aspirate (Online Supplementary Figure S2B). Notably, pAML sensitivity to killing did not depend on whether the sample was acquired at onset or at relapse. Although our sample set was limited, we observed that six of the seven pAML samples with corebinding factor (CBF) rearrangements (inv(16)(CBFBMYH11) and t(8;21)(RUNX1-RUNX1T1)), which are associated with a more favorable prognosis, ${ }^{4}$ were classified as IR or R.
Killing-sensitive pediatric acute myeloid leukemia (pAML) have significantly different gene expression than resistant pAML

In order to identify factors impacting pAML sensitivity to LV-10 killing, we performed RNA-seq on 14 S, IR, and R pAML. We found 335 differentially expressed genes (DEG) between $S$ and R pAML (absolute $\log _{2}$ fold change $[\mathrm{FC}] \geq 2$, false discovery rate $[\mathrm{FDR}]<0.05$ ) (Figure 2A; Online Supplementary Table S2). Between the other groups, we found 247 DEG between the S and IR pAML, while the IR and R pAML were more similar, with only 27 DEG, (Online Supplementary Figure S3A and B; Online Supplementary Tables S3 and 4). 
A Heat map of DEG between $S$ and $R$ pAML

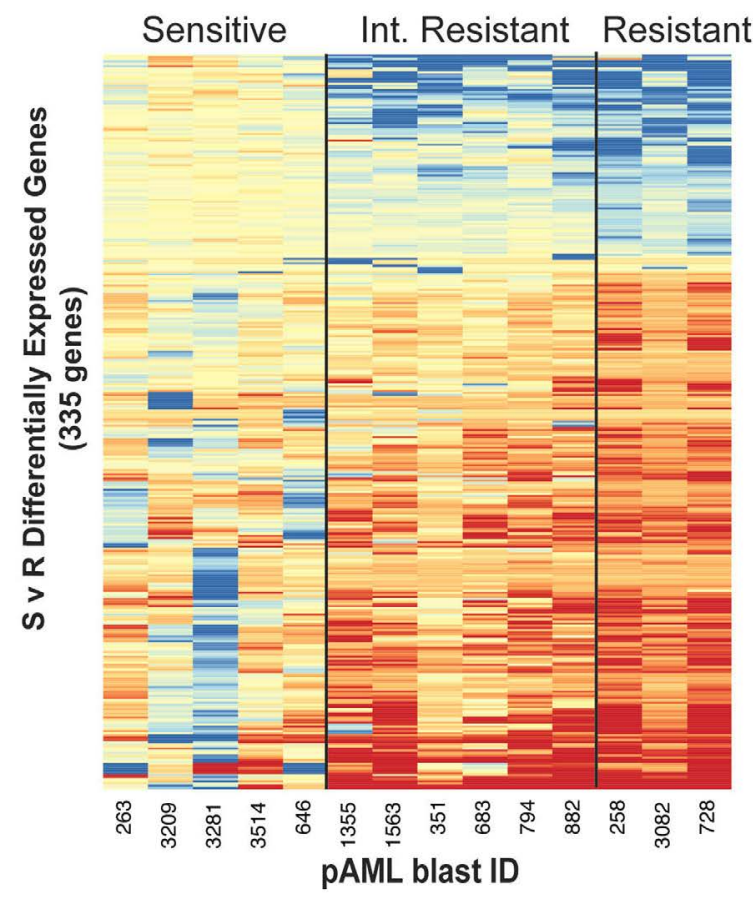

\section{B GO Terms Enriched in Sensitive}

Scaled to FDR q-val

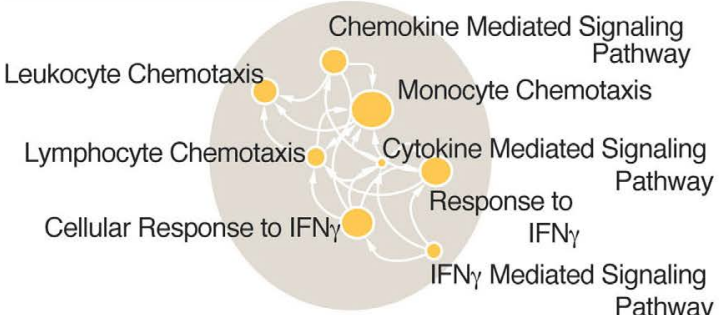

C Myeloid marker expression

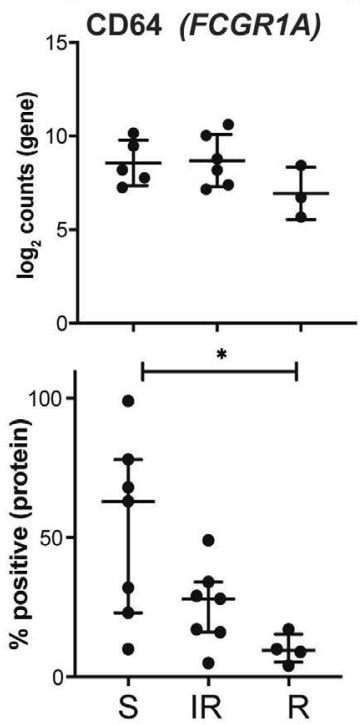

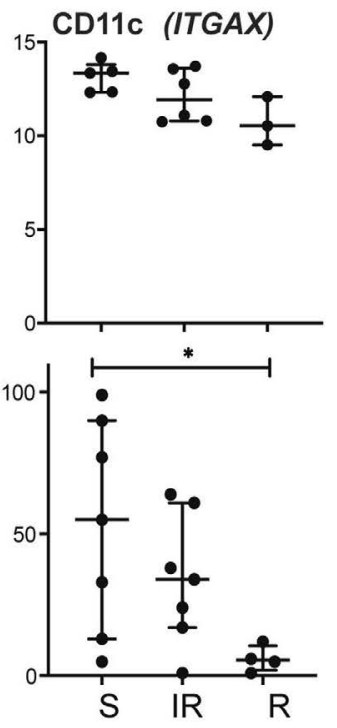

Figure 2. Sensitive and resistant pediatric acute myeloid leukemia have distinct gene expression profiles. (A) Two-dimensional heatmap of differentially expressed genes (DEG) identified between sensitive (S) and resistant (R) pediatric acute myeloid leukemia (pAML). DEG with a false discovery rate (FDR) $<0.05$ and absolute $\left(\log _{2}\right.$ fold change $\left.[F C]\right) \geq 2$ were identified using differential gene expression analysis (DESeq2). Gene expression values were normalized with respect to the sensitive pAML group, by subtracting the mean expression of sensitive pAML. (B) Gene set enrichment in sensitive pAML. Gene ontology (GO) term enrichment was performed using Gene Set Enrichment Analysis and GO terms with FDR $q<0.2$ were visualized using EnrichmentMap in Cytoscape. Circle size is inversely scaled to the FDR $q$ value. (C) Sensitive pAML have high expression of mature myeloid markers. Top panels show bulk RNA sequencing (RNA-seq) log ${ }_{2}$ counts, and the bottom panels show the frequency of pAML expressing the indicated proteins, measured using clinical flow cytometry gated on blast cells. Error bars: median and interquartile range. ${ }^{*} P<0.05$. IR: intermediate resistant.

We next examined GO term enrichment in sensitive and resistant pAML using Gene Set Enrichment Analysis (GSEA). ${ }^{30,31}$ We visualized the results using EnrichmentMap ${ }^{28}$ to collapse the GO terms into sub-clusters. Sensitive pAML showed strong signatures of IFN- $\gamma$ related genes and monocyte chemotaxis (Figure 2B; Online Supplementary Table S5). We also observed that the protein expression of monocytic genes (CD64, CD11c, CD4, CD15, and CD33) largely contributed to the observed variance amongst the clinical flow cytometry phenotypes of pAML samples (Online Supplementary Figure S4). In order to investigate this monocytic signature, we visualized the gene expression of selected, established AML maturation markers ${ }^{4}$ from the RNA-seq data (Figure 2C, top) derived from the bulk bone marrow aspirate lysates, and matched it to the corresponding proteins expressed on pAML blasts, measured by clinical flow cytometry phenotyping (Figure 2C, bottom). CD11c and CD64 proteins, which are commonly observed in mature, monocytic $\mathrm{AML}^{32,33}$ were expressed significantly higher in sensitive than in resistant pAML blasts (Figure 2C).
Pediatric acute myeloid leukemia (pAML) sensitivity and resistance signatures observed in NCI TARGET pAML transcriptome dataset

In order to determine if the gene expression signatures of sensitivity and resistance we observed in our pAML samples can be found in a larger cohort, we analyzed our dataset together with a 187-sample NCI TARGET pAML dataset, the largest comprehensive pAML dataset publicly available. ${ }^{19}$ Principal component analysis on the most variant genes showed that the Stanford pAML samples distributed among the TARGET pAML samples, indicating that the sample source was not a dominant technical covariate (Online Supplementary Figure S5A). Unsupervised analysis of the combined Stanford and TARGET pAML datasets confirmed that Stanford pAML samples did not cluster independently (Online Supplementary Figure S5B). Interestingly, out of the four major clusters, two clusters contained only the S pAML while the other two clusters contained both the IR and R pAML.

Next, we examined if the 335-gene signature discriminating between $\mathrm{S}$ and $\mathrm{R}$ pAML was present in the TARGET dataset. Clustering of the combined pAML dataset based 


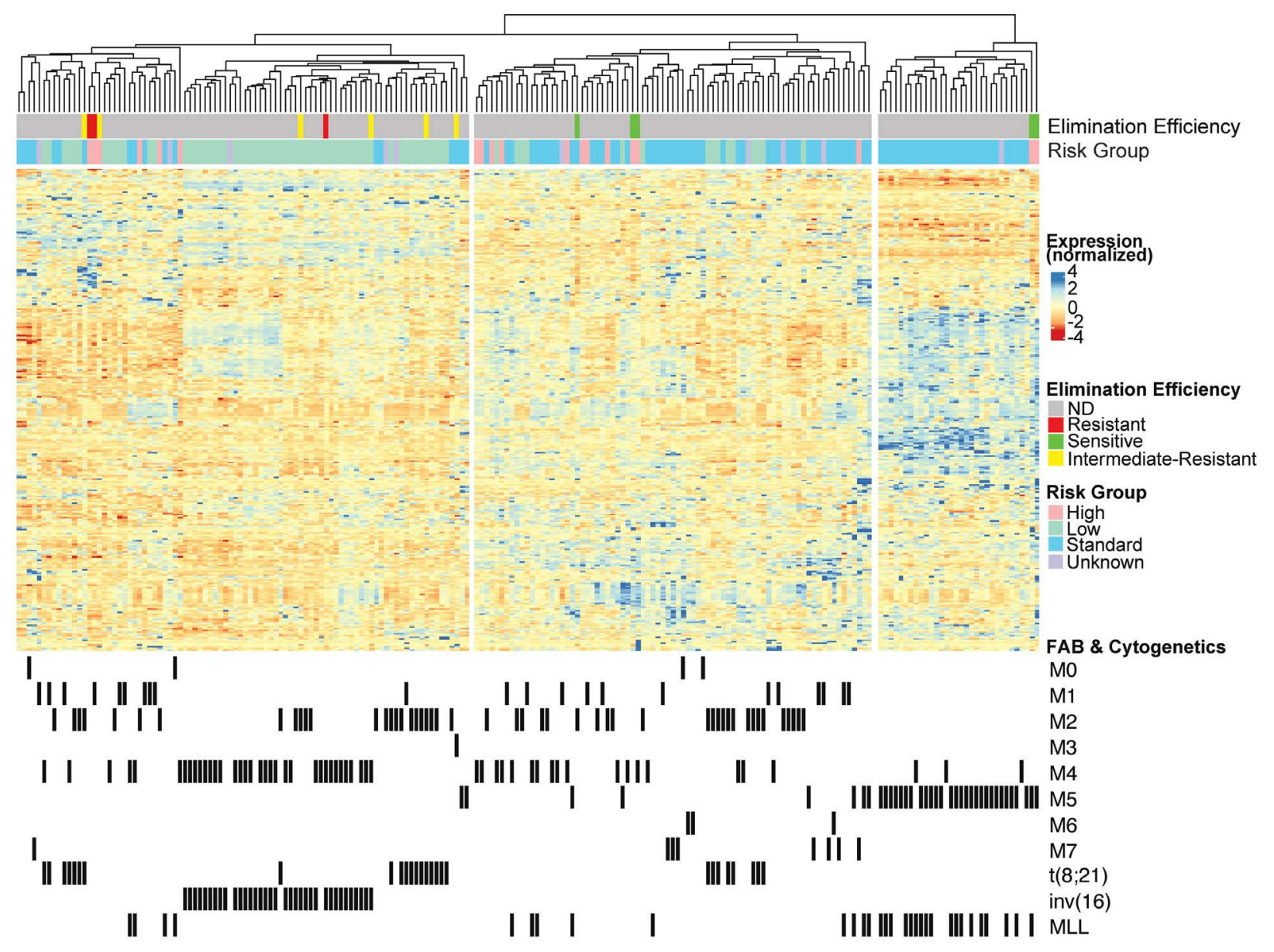

Figure 3. Sensitive and resistant pediatric acute myeloid leukemia (pAML) signatures group TARGET pAML into three clusters. Euclidean clustering of the differentially expressed genes (DEG) between sensitive and resistant pediatric acute myeloid leukemia (pAML) (false discovery rate [FDR] <0.05, and absolute (log fold change $[F C]$ ) $\geq 2$ ) detected in sequencing data from our Stanford dataset and the Therapeutically Applicable Research to Generate Effective Treatments (TARGET) PAML dataset. Measured LV-10 killing sensitivity, risk group, and French-American-British (FAB) diagnosis are matched to each pAML when applicable. The TARGET dataset does not have associated LV-10 killing assay outcomes. Expression color is scaled per gene row. ND: not determined.

on their expression of the identified DEG grouped the samples into three primary clusters: two 'sensitive' clusters that grouped with S pAML and $57 \%$ of TARGET pAML, and a 'resistant' cluster that grouped with the IR and R pAML and $43 \%$ of TARGET pAML (Figure 3 ). As we observed that CBF pAML were highly represented in IR and R pAML, we examined their distribution in the combined Stanford-TARGET dataset. Both pAML with t(8;21)(RUNX1-RUNX1T1) and pAML with inv(16)(CBFB-MYH11) translocations were enriched in the 'resistant' cluster $(P<0.0001, P<0.0001$ respectively). In line with our GSEA analysis results, one of the 'sensitive' clusters was highly enriched for M5 monocytic pAML $(P<0.0001)$, while the 'resistant' cluster was enriched for M4 myelomonocytic pAML that also displayed rearrangement inv(16) $(P<0.0001)$.

\section{Resistant pediatric acute myeloid leukemia express high levels of CD200, which can impair LV-10 cytotoxicity}

In order to identify genes linked with pAML sensitivity or resistance to LV-10 cell killing, we correlated gene expression to the median elimination efficiency for each pAML blast. The expression of 2,181 genes significantly correlated to killing with $P<0.05$ (Figure 4A; Online Supplementary Table S6), 395 of which had an absolute $R \geq 0.7$ (Figure $4 A$, genes shown as red bars). We hypothesized that the resistant pAML expressed inhibitory markers that protected them from killing. Therefore, we overlaid the genes that significantly and negatively correlated with killing, R $\leq-0.7$ (189 genes), with the genes that were overexpressed 4-fold or more in the resistant pAML from the DEG analysis of sensitive versus resistant pAML (899 genes). We found 60 genes that were both negatively correlated with killing and preferentially expressed in resistant pAML (Figure 4B). Since perforin and granzyme B-mediated killing requires cell-to-cell interaction, ${ }^{17,34}$ we filtered this gene list for genes encoding surface proteins $s^{35}$ and identified 10 genes (Figure 4B).

Next, we manually examined the functions of the ten genes to uncover proteins that have known interacting receptors expressed on $T$ cells, and identified CD200, a type 1 membrane glycoprotein. CD200 is upregulated on resistant pAML (Figure 4C and D), and LV-10 express the CD200 receptor (CD200R1) (Figure 4E), an inhibitory receptor of immunoglobulin superfamily. ${ }^{36} \mathrm{CD} 200$ expression is associated with poor prognosis in adult AML. ${ }^{24,25}$ Moreover, CD200R1 signaling has been previously shown to impair mast cell ${ }^{13}$ and $\mathrm{CD}^{+} \mathrm{T}$-cell degranulation. ${ }^{38}$

In order to determine if CD200 expression confers resistance to LV-10-mediated killing, we overexpressed CD200 in killing-sensitive ALL-CM and U937 myeloid cell lines. For 
A Correlation between E.E. and gene expression

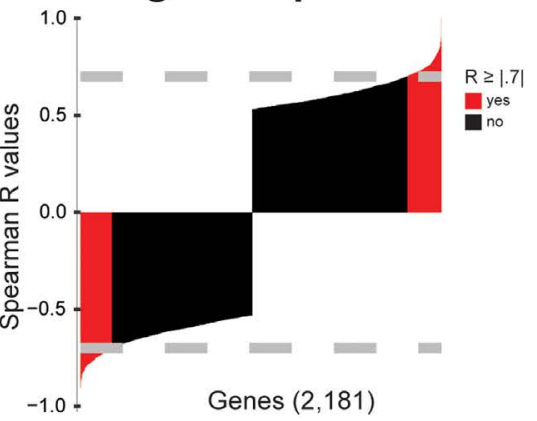

C CD200 gene expression

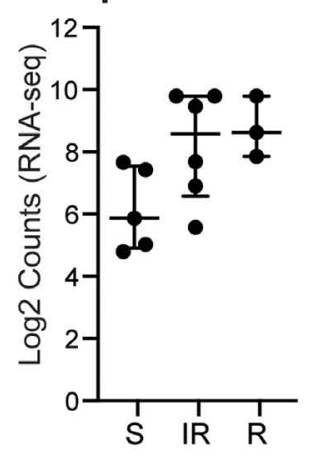

D CD200 protein expression

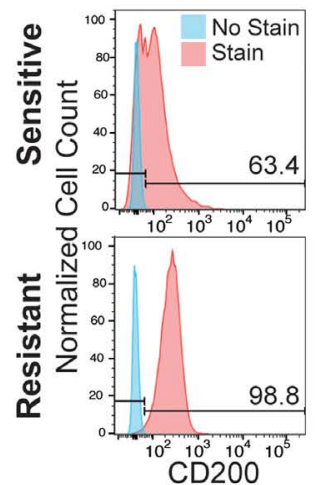

F LV-10 degranulation in co-culture

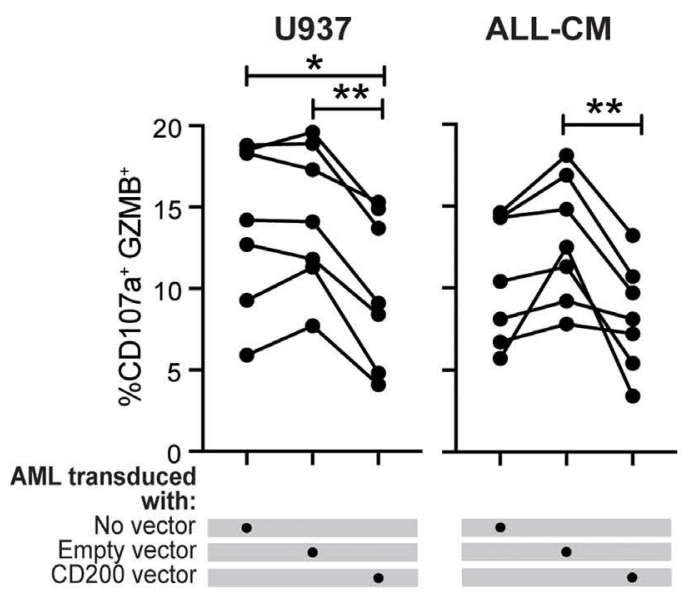

Identification of surface markers associated with pAML killing

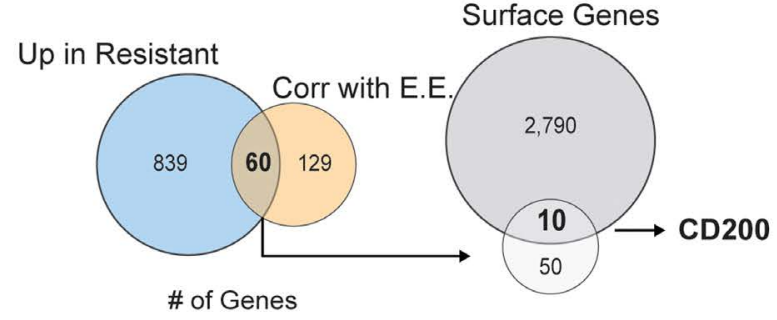

E CD200R1 protein on LV lines

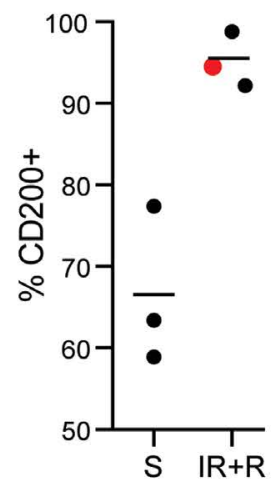

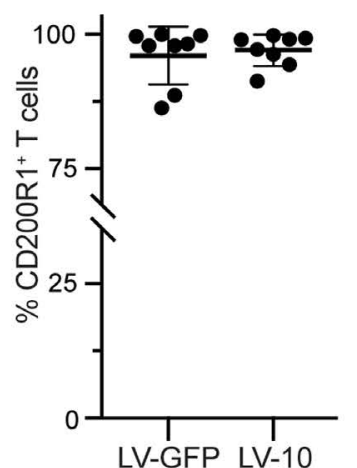

G LV-10 degranulation with U937 ${ }^{\mathrm{CD} 200}$

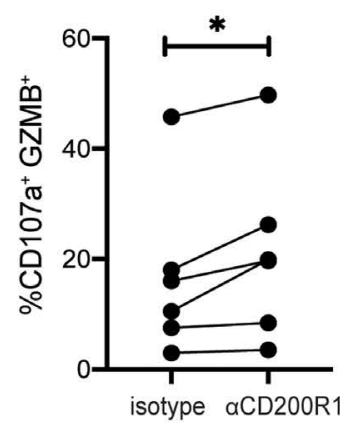

H Target cell killing by LV-10

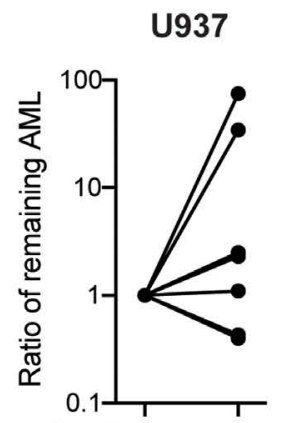

ALL-CM

AML transduced

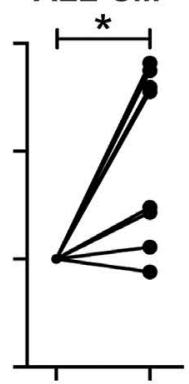


Figure 4. CD200 expression is upregulated in resistant pediatric acute myeloid leukemia and can impair LV-10-mediated degranulation and cytotoxicity. (A) Expression of 395 genes positively or negatively correlating with pediatric acute myeloid leukemia (pAML) sensitivity. The Spearman correlation of the expression of each gene to the median elimination efficiency (E.E.) of each pAML was calculated and plotted with genes represented as bars. 2,181 genes had a correlation with $P<0.05,395$ of which had an absolute $(R) \geq 0.7$ (red bars). (B) Data-mining strategy to identify genes conferring pAML resistance to LV-10 killing. Genes expressed 4 -fold or more in the resistant pAML from the differentially expressed gene (DEG) analysis between sensitive vs resistant and the list of genes negatively correlated with E.E. with $P<0.05$ and $\mathrm{R} \leq-0.7$ were used to identify overlap in a Venn diagram. Genes appearing in both enriched in resistant and negatively correlated with E.E. were overlaid with genes encoding surface proteins identified in the Cell Surface Protein Atlas, ${ }^{35}$ resulting in ten pAML genes encoding surface proteins. These genes were manually annotated for potential interaction with T-cell surface proteins, identifying CD200. (C) CD200 gene expression in pAML blasts; log ${ }_{2}$ counts. Error bars: median and interquartile range. (D) CD200 protein expression on pAML, flow cytometry. Left panel: representative plots for one sensitive and one resistant pAML blast; right panel: cumulative data. Values from resistant (R) and intermediate resistant (IR) pAML are grouped, with IR pAML in red. Line represents mean. (E) CD200 receptor (CD200R1) is expressed on both LV-10 and LV-GFP cells. CD200R1 expression was measured in the CD $3^{+} \mathrm{CD}^{+}{ }^{+} \mathrm{NGFR}{ }^{+}$population by flow cytometry, $\mathrm{n}=8$. Line represents mean; error bar standard deviation. (F) CD200 overexpression impairs LV-10 degranulation. LV-10 were co-cultured with target myeloid tumor cell lines at a 10:1 E:T ratio for 6 hours. LV-10 cell line $(n=7)$ degranulation, as measured by \%CD107a ${ }^{+} \mathrm{GZMB}^{+}$co-expression, was determined in co-culture with untransduced U937 and ALL-CM cells (No Vector), sorted GFP ${ }^{+}$U937 and ALL-CM cells transduced with an empty vector (Empty Vector), or sorted GFP CD200 ${ }^{+}$U937 and ALL-CM cells transduced with CD200 (CD200 Vector). * $P<0.05, * * P<0.01$, Friedman ANOVA with Dunn's post hoc test. (G) Cumulative CD107a ${ }^{+}$GZMB ${ }^{+} \%$ degranulation of LV-10 against U937 overexpressing CD200 when pre-treated with isotype control or anti-CD200R1 blocking antibody prior to co-culture, $\mathrm{n}=6$; $* P<0.05$, Wilcoxon test. (H) CD200 reduces LV-10-mediated killing. LV-10 cells were co-cultured with empty vector- or CD200-overexpressing U937 or ALL-CM cells at a 1:1 E:T ratio for 3 days. Surviving cells were enumerated by flow cytometry. Ratio of remaining pAML was calculated as: (\# remaining cells in CD200-overexpressing AML $+\mathrm{LV}-10$ /\# remaining cells in CD200-overexpressing $\mathrm{AML}$ alone)/(\# remaining cells in empty vector- $\mathrm{AML}+\mathrm{LV}-10 / \#$ remaining cells in empty vector $\mathrm{AML}$ alone). ( $\mathrm{n}=8$, $\star P<0.05$, Wilcoxon test.) GZMB: granzyme B.

this, we constructed a bicistronic lentiviral vector containing CD200 together with ZsGreen1, a green fluorescent protein (Online Supplementary Figure S6A). Both cell lines transduced with the CD200-containing vector displayed significant upregulation of CD200 protein compared to empty vector-transduced cells (Online Supplementary Figure $S 6 B)$. First, we tested the impact of CD200R1 signaling on LV-10 degranulation using CD107a degranulation assay coupled with granzyme B intracellular staining. ${ }^{39}$ In comparison to the LV-10 cells co-cultured with control cell lines, LV-10 co-cultured with CD200-overexpressing cell lines degranulated significantly less (Figure 4F). In order to test if we could rescue the reduction in degranulation induced by CD200-overexpressing cells, we blocked CD200R1 on the LV-10 with a neutralizing antibody prior to co-culture with U937 or U937 CD200-overexpressing cell lines (Online Supplementary Figure S7A). Blocking CD200R1 partially restored LV-10 degranulation when co-cultured with CD200-overexpressing U937 (Figure 4G), while it had a non-significant effect on LV-10 degranulation when co-cultured with wild-type U937 (Online Supplementary Figure S7B). LV-10 degranulation was not fully restored to levels induced by wild-type U937, likely because the CD200R1 neutralizing antibody only blocked approximately $50 \%$ of available CD200R1 (Online Supplementary Figure S7C).

We next tested if CD200 overexpression in myeloid leukemia cell lines could confer resistance to LV-10 killing. In comparison to the empty vector-transduced control cells, CD200 overexpression significantly reduced killing of ALLCM cells, but not of U937 cells (Figure 4H). This may be due to U937 cells' increased robustness in vitro, as they have an average 1.34-fold higher proliferation rate than ALL-CM cells (not shown) that could compensate for killing in a 3-day culture. LV-GFP degranulation and killing, which are less potent than in LV-10 cells (Online Supplementary Figure S8), was also impaired by CD200, indicating that the CD200R1 signaling-induced inhibition of cytotoxicity is not Tr1-specific. Altogether, these data suggest that resistant pAML can evade LV-10 killing by impairing their degranulation via CD200 expression.

\section{Discussion}

AML is a highly diverse hematopoietic cancer with over 20 different WHO sub-classifications, ${ }^{29}$ with suboptimal responses to conventional therapy and an urgent need for novel treatments. ${ }^{40}$ Our previous study revealed that four of eight primary adult AML were sensitive to LV-10 cell killing. Importantly, LV-10 cells could inhibit myeloid leukemia progression in vivo while preventing the induction of GvHD when co-injected with $\mathrm{CD} 4^{+} \mathrm{T}$ cells, ${ }^{13}$ suggesting that LV-10 cells can represent an innovative cell therapy for AML. Since pAML differ substantially from adult AML at the molecular, epigenetic, and genetic levels, ${ }^{19-23}$ herein we determined the pAML sensitivity to LV-10 killing, characterized the sensitive and resistant pAML molecular profiles, and identified CD200 expression as one of the mechanisms of pAML resistance to LV-10 killing.

While previously tested adult AML had only two levels of sensitivity to LV-10 killing, ${ }^{13}$ resembling the intermediate resistant and resistant pAML we measured, we also observed a subset of pAML that were highly sensitive to elimination by LV-10 cells. This additional sensitivity category may reflect the intrinsic genetic and epigenetic differences between adult and pediatric AML, ${ }^{19-23}$ which could affect expression of markers required for LV-10-mediated killing. Interestingly, we observed that the expression of CD13, CD54, or CD112, which positively correlated with sensitivity to LV-10-mediated killing in adult AML, ${ }^{13}$ did not correlate to pAML sensitivity to killing (data not shown), further supporting the hypothesis that pediatric and adult AML interact differently with LV-10 cells.

The range of sensitivities we observed in pAML was underscored by significant differences in gene expression and cytogenetics. These analyses revealed that sensitivity to killing was linked to differentiation status. Sensitive pAML resembled more mature differentiated myeloid cells, with an enrichment of monocytic genes and high levels of CD64 and CD11c protein, which are frequently described on more differentiated AML subtypes. ${ }^{32,33,41,42}$ Conversely, resistant pAML did not have as distinct a gene signature. We found the maturation signature we observed in our sensitive subset present in the 187-sample NCI TARGET-pAML dataset. ${ }^{19}$ Whether we clustered the combined Stanford and TARGET data sets using only the top variably expressed genes or with our filtered $S \mathrm{v} R$ DEG list, the S pAML independently clustered away from the IR/R pAML. This was partially because the top $10 \%$ variability expressed genes in the combined Stanford and TARGET pAML datasets incorporated around half of the 335 DEG discriminating S v R pAML, yet this also suggests that the $S \vee R$ DEG may represent underlying distinguishing features among pAML. In addition to the genes driving the clustering, cytogenetic abnormalities, specifi- 
cally the core binding factor translocations $\mathrm{t}(8 ; 21)$ (RUNX1-RUNX1T1) and inv(16), were consistently overrepresented within the IR and R pAML containing cluster. Despite the typically more favorable prognosis for pAML with core binding factor translocations, ${ }^{4}$ these pAML could evade T-cell killing in vitro. While the role of core binding factor translocations in immune evasion is not well understood, it has been observed these lesions can impair naural killer (NK) cell surveillance of target cells through downregulation of CD48, an NK cell ligand. ${ }^{43}$ Conversely, in the S containing cluster, there was enrichment of patients with MLL rearrangements that historically have intermediate to poor outcome. MLL rearrangements account for $15-20 \%$ of all pAML cases, ${ }^{44}$ but only $3 \%$ of adult $\mathrm{AML},{ }^{45}$ which suggest that LV-10 cells may be uniquely suited for the treatment of a common pAML subset. Further analysis of the sensitivity of specific subsets of pAML to LV-10 mediated killing may improve our ability to identify key genes responsible for the sensitivity of these pAML subsets.

We also identified CD200 as upregulated on IR and R pAML. CD200 has previously been associated with poor patient outcomes in adult AML. ${ }^{24,25}$ CD200 is a membrane glycoprotein that induces an inhibitory signal upon binding to its cognate inhibitory receptor CD200R1, and impairs degranulation in mast cells and $\mathrm{CD}^{+} \mathrm{T}$ cells. ${ }^{37,38} \mathrm{CD} 200 \mathrm{R} 1$ is expressed on both LV-10 and control LV-GFP cells. CD200 has negligible baseline expression on killing-sensitive ALLCM, U937, and THP-1 myeloid cell lines (data not shown). We found that the overexpression of CD200 on ALL-CM and U937 cell lines led to a significant impairment in LV-10 degranulation, and in one cell line, CD200 overexpression also increased AML survival in the killing assay. CD200 effect on LV-10 degranulation was specific to CD200/CD200R1 interaction, as the degranulation increased upon CD200R1 blockade. Interestingly, CD200 expression also impaired the response of the Teff-like control LV-GFP cells. These results, together with reports showing that CD200 expression on AML can impair CD8 ${ }^{+} \mathrm{T}$-cell function, ${ }^{38}$ support the role of CD200 signaling in the impairment of cytotoxic T-cell degranulation. Notably, LV-10-mediated degranulation and killing of CD200-overexpressing AML cell lines were only impaired, not completely abolished, again suggesting that resistant pAML express multiple genes that contribute to their evasion of LV-10 killing.

Our observation that LV-10 cells can eliminate a large subset of pAML, together with our previously published data showing their ability to eliminate AML cell lines in vivo, support their use as a novel therapy for high-risk pAML patients receiving allo-HSCT. ${ }^{13}$ We foresee the two potential uses of LV-10 cells in the clinic. First, donor-derived LV-10 cells could be used alongside allo-HSCT, acting early to prevent GvHD and combat residual AML. Alternatively, LV-10 can be used for their GvL effect when the patients' own immune cells are depleted. Patients who are minimal residual disease positive after induction chemotherapy have an abysmal prognosis, with only $10 \%$ disease-free survival. ${ }^{46}$ In those patients, LV-10 cells could be used as a less toxic alternative to another round of induction chemotherapy prior to alloHSCT. LV-10 would eliminate residual AML blasts, while persisting 2-3 weeks in vivo without eliciting GvHD (our unpublished data in humanized mice), until the patient's own immune system reconstitutes. Notably, to mediate killing, LV-10 cells do not need to recognize specific antigens on their target cells through the TCR, ${ }^{13,18}$ uncoupling their cytotoxicity from HLA-II match or mismatch. We are in the process of further investigating mechanisms of LV-10 recognition of AML, and their potential therapeutic applications with in vivo models, in preparation for the transition of LV10 cell therapy to the clinic.

In conclusion, we show that LV-10 cells can directly mediate killing of pAML, especially those with an activated, mature myeloid gene expression profile. $\mathrm{pAML}$ resistance to killing was associated with expression of CD200, an immunomodulatory protein associated with poor AML prognosis, ${ }^{25}$ which could impair LV-10 cytotoxic responses. It is possible that by blocking the effect of these resistance factors, we may reverse resistance to LV-10-mediated killing. Altogether, our previous work and current findings imply that LV-10 cell therapy might be well suited to treat pAML by providing both a GvL effect and preventing GvHD, thus improving the outcome for many children with high risk pAML.

\section{Disclosures \\ No conflicts of interest to disclose.}

\section{Contributions}

$B C, M J U, S G, N J L, A M C$ and MGR designed the research; $B C, M J U, P C, J M L, K G$ and ECS performed experiments; $B C$, $M J U, P C, G A, R B, A B, A M C$ and $M G R$ analyzed the data; $B C$, $M J U, A B, N J L, A M C$ and $M G R$ wrote the paper.

\section{Acknowledgments}

We would like to thank the Bass Center Tissue Bank staff members for preparing patient samples and data, and Drs. Ravindra Majeti, Tian Zhang, Yusuke Nakauchi, Daniel Thomas, Robertson Parkman, and Rhonda Perriman for helpful discussions, and Drs. Nicole Baldwin, Jacob Cardenas, Jinghua Gu, as well as Cynthia Smitherman and Phuong Nguyen from the Genomics and Biostatistics Cores of the Baylor Institute for Immunology Research in Dallas, TX, for RNA sample processing, RNA sequencing and initial data analysis.

\section{Funding}

This work was supported by a Rising Tide Foundation for Clinical Cancer Research, Curesearch for Children's Cancer Acceleration Initiative grant, Emerson Collective Cancer Research Fund, Alex's Lemonade Stand Reach Grant, Children's Leukemia Research Association, the CURE Childhood Cancer Foundation, and the Virginia and D.K. Ludwig Fund for Cancer Research. BC was supported by the Maternal and Child Health Research Institute Postdoctoral Fellowship. MJU was supported by an NSF DGE (1147470) and Blavatnik Family Fellowship.

\section{References}

1. Vedi A, Mitchell R, Shanmuganathan S, et al. Increased survival for children with acute myeloid leukemia results from improved postrelapse treatment. J Pediatr Hematol
Oncol. 2018;40(7):541-547.

2. Ravindranath Y, Chang M, Steuber CP, et al. Pediatric Oncology Group (POG) studies of acute myeloid leukemia (AML): a review of four consecutive childhood AML trials conducted between 1981 and 2000. Leukemia. 2005;19(12):2101-2116.
3. Sander A, Zimmermann M, Dworzak M, et al. Consequent and intensified relapse therapy improved survival in pediatric AML: results of relapse treatment in 379 patients of three consecutive AML-BFM trials. Leukemia. 2010;24(8):1422-1428.

4. Creutzig U, van den Heuvel-Eibrink MM, 
Gibson B, et al. Diagnosis and management of acute myeloid leukemia in children and adolescents: recommendations from an international expert panel. Blood. 2012;120(16):3187-3205.

5. Yi T, Chen Y, Wang L, et al. Reciprocal differentiation and tissue-specific pathogenesis of Th1, Th2, and Th17 cells in graft-versushost disease. Blood. 2009;114(14):31013112 .

6. Wingard JR, Majhail NS, Brazauskas R, et al. Long-term survival and late deaths after allogeneic hematopoietic cell transplantation. J Clin Oncol. 2011;29(16):2230-2239.

7. Kumar S, Chen M, Gastineau D, et al. Effect of slow lymphocyte recovery and type of graft-versus-host disease prophylaxis on relapse after allogeneic bone marrow transplantation for acute myelogenous leukemia. Bone Marrow Transplant. 2001;28(10):951956.

8. Groux H, O'Garra A, Bigler M, et al. A CD4+T-cell subset inhibits antigen-specific T-cell responses and prevents colitis. Nature. 1997;389(6652):737-742

9. Blazar BR, MacDonald KPA, Hill GR. Immune regulatory cell infusion for graftversus-host disease prevention and therapy. Blood. 2018;131(24):2651-2660

10. Roncarolo MG, Gregori S, Bacchetta R, Battaglia M, Gagliani N. The biology of T regulatory type 1 cells and their therapeutic application in immune-mediated diseases. Immunity. 2018;49(6):1004-1019.

11. Bacchetta R, Lucarelli B, Sartirana C, et al. Immunological outcome in haploidenticalHSC transplanted patients treated with IL10-anergized donor T cells. Front Immunol. 2014;5:16.

12. Serafini G, Andreani M, Testi M, et al. Type 1 regulatory $\mathrm{T}$ cells are associated with persistent split erythroid/lymphoid chimerism after allogeneic hematopoietic stem cell transplantation for thalassemia. Haematologica. 2009:94(10):1415-1426.

13. Locafaro G, Andolfi G, Russo F, et al. IL-10engineered human CD4 + Tr1 cells eliminate myeloid leukemia in an HLA class Idependent mechanism. Mol Ther. 2017; 25(10):2254-2269.

14. Andolfi G, Fousteri G, Rossetti M, et al. Enforced IL-10 expression confers type 1 regulatory $\mathrm{T}$ cell (Tr1) phenotype and function to human CD4+ T cells. Mol Ther. 2012;20(9):1778-1790.

15.Zeller JC, Panoskaltsis-Mortari A, Murphy WJ, et al. Induction of CD4+ T cell alloantigen-specific hyporesponsiveness by IL-10 and TGF-beta. J Immunol. 1999;163(7):36843691.

16. Zhang P, Lee JS, Gartlan KH, et al. Eomesodermin promotes the development of type 1 regulatory $T\left(\begin{array}{lll}T & R & 1\end{array}\right)$ cells. Sci Immunol. 2017;2(10):eaah7152

17. Magnani CF, Alberigo G, Bacchetta R, et al. Killing of myeloid APCs via HLA class I, CD2 and CD226 defines a novel mechanism of suppression by human Tr1 cells. Eur J Immunol. 2011;41(6):1652-1662.

18. Guipouy D, Gertner-Dardenne J, Pfajfer L, German Y, Belmonte N, Dupré L. Granulysin- and granzyme-dependent elim- ination of myeloid cells by therapeutic ovaspecific type 1 regulatory $\mathrm{T}$ cells. Int Immunol. 2019:31(4):239-250

19. Bolouri H, Farrar JE, Triche T, et al. The molecular landscape of pediatric acute myeloid leukemia reveals recurrent structural alterations and age-specific mutational interactions. Nat Med. 2017;24(1):103-112.

20. Ho PA, Kutny MA, Alonzo TA, et al. Leukemic mutations in the methylationassociated genes DNMT3A and IDH2 are rare events in pediatric AML: a report from the Children's Oncology Group. Pediatr Blood Cancer. 2011;57(2):204-209.

21. Radtke I, Mullighan CG, Ishii $M$, et al. Genomic analysis reveals few genetic alterations in pediatric acute myeloid leukemia. Proc Natl Acad Sci U S A. 2009; 106(31): 12944-12949.

22. Marceau-Renaut A, Duployez N Ducourneau B, et al. Molecular profiling defines distinct prognostic subgroups in childhood AML: a report from the French ELAM02 Study Group. HemaSphere. 2018; 2(1):e31.

23. Juhl-Christensen C, Ommen HB Aggerholm A, et al. Genetic and epigenetic similarities and differences between childhood and adult AML: molecular profiling in childhood AML. Pediatr Blood Cancer. 2012;58(4):525-531.

24. Tonks A, Hills R, White P, et al. CD200 as a prognostic factor in acute myeloid eukaemia. Leukemia. 2007;21(3):566-568.

25. Tiribelli M, Geromin A, Cavallin M, et al. ABCG2 and CD200 define patients at high risk of relapse in ELN favorable subgroup of AML. Eur J Haematol. 2017;99(3):269-274.

26. Love MI, Huber W, Anders S. Moderated estimation of fold change and dispersion for RNA-seq data with DESeq2. Genome Biol. 2014;15(12):550.

27. Gu Z, Eils R, Schlesner M. Complex heatmaps reveal patterns and correlations in multidimensional genomic data. Bioinformatics. 2016:32(18):2847-2849.

28. Merico D, Isserlin R, Stueker O, Emili A, Bader GD. Enrichment map: a networkbased method for gene-set enrichment visualization and interpretation. PLoS One. 2010;5(11):e13984.

29. Arber DA, Orazi A, Hasserjian R, et al. The 2016 revision to the World Health Organization classification of myeloid neoplasms and acute leukemia. Blood. 2016;127(20):2391-2405.

30. Mootha VK, Lindgren CM, Eriksson K-F, et al. PGC-1 $\alpha$-responsive genes involved in oxidative phosphorylation are coordinately downregulated in human diabetes. Nat Genet. 2003;34(3):267-273

31. Subramanian A, Tamayo P, Mootha VK, et al. Gene set enrichment analysis: a knowledge-based approach for interpreting genome-wide expression profiles. Proc Natl Acad Sci U S A. 2005:102(43):15545-15550.

32. Dunphy CH, Tang W. The value of CD64 expression in distinguishing acute myeloid leukemia with monocytic differentiation from other subtypes of acute myeloid leukemia: a flow cytometric analysis of 64 cases. Arch Pathol Lab Med. 2007;
131(5):748-754

33. Krasinskas AM, Wasik MA, Kamoun M, Schretzenmair R, Moore J, Salhany KE. The usefulness of CD64, other monocyte-associated antigens, and CD45 gating in the subclassification of acute myeloid leukemias with monocytic differentiation. Am J Clin Pathol. 1998;110(6):797-805

34. Jenkins MR, Griffiths GM. The synapse and cytolytic machinery of cytotoxic $\mathrm{T}$ cells. Curr Opin Immunol. 2010;22(3):308-313.

35. Bausch-Fluck D, Goldmann U, Müller S, et al. The in silico human surfaceome. Proc Natl Acad Sci U S A. 2018;115(46):E10988E10997.

36. Vaine CA, Soberman RJ. The CD200CD200R1 inhibitory signaling pathway. In Advances in Immunology. Elsevier; p191211

37. Cherwinski HM, Murphy CA, Joyce BL, et al. The $\mathrm{CD} 200$ receptor is a novel and potent regulator of murine and human mast cell function. J Immunol. 2005;174(3):13481356.

38. Coles SJ, Hills RK, Wang ECY, et al Expression of CD200 on AML blasts directly suppresses memory T-cell function. Leukemia. 2012;26(9):2148-2151.

39. Betts MR, Brenchley JM, Price DA, et al. Sensitive and viable identification of antigen-specific CD8+ $\mathrm{T}$ cells by a flow cytometric assay for degranulation. J Immunol Methods. 2003;281(1-2):65-78.

40. Winer ES, Stone RM. Novel therapy in Acute myeloid leukemia (AML): moving toward targeted approaches. Ther Adv Hematol. 2019:10:204062071986064.

41. Gorczyca W, Sun Z-Y, Cronin W, Li X, Mau S, Tugulea S. Immunophenotypic pattern of myeloid populations by flow cytometry analysis. In: Methods in Cell Biology. Elsevier; p221-266.

42. Raspadori D, Damiani D, Lenoci $M$, et al. CD56 antigenic expression in acute myeloid leukemia identifies patients with poor clinical prognosis. Leukemia. 2001;15(8):11611164

43. Elias S, Yamin R, Golomb L, et al. Immune evasion by oncogenic proteins of acute myeloid leukemia. Blood. 2014; 123(10): 1535-1543.

44. Balgobind BV, Zwaan CM, Pieters R, Van den Heuvel-Eibrink MM. The heterogeneity of pediatric MLL-rearranged acute myeloid leukemia Leukemia. 2011:25(8):1239-1248.

45. Schoch C, Schnittger S, Klaus M, Kern W, Hiddemann W, Haferlach T. AML with 11q23/MLL abnormalities as defined by the WHO classification: incidence, partner chromosomes, FAB subtype, age distribution, and prognostic impact in an unselected series of 1897 cytogenetically analyzed AML cases. Blood. 2003;102(7):2395-2402.

46. Jacobsohn DA, Loken MR, Fei $M$, et al. Outcomes of measurable residual disease in pediatric acute myeloid leukemia before and after hematopoietic stem cell transplant: validation of difference from normal flow cytometry with chimerism studies and Wilms tumor 1 gene expression. Bio Blood Marrow Transplant. 2018;24(10) 2040-2046 